\title{
Complex fuzzy soft matrices with applications
}

\author{
Madad Khan*1 (D), Saima Anis ${ }^{1}$ (D), Seok-Zun Song ${ }^{2,3}$ (D), Young Bae Jun ${ }^{4}$ (iD \\ ${ }^{1}$ Department of Mathematics, COMSATS University Islamabad, Abbottabad Campus, Pakistan \\ ${ }^{2}$ Department of Mathematics, Jeju National University, Jeju 63243, Korea \\ ${ }^{3}$ School of Computational Sciences, Korean Institute for Advanced Study, Seoul, 02455, Korea \\ ${ }^{4}$ Department of Mathematics Education, Gyeongsang National University, Jinju, 52828, Korea
}

\begin{abstract}
In this paper, we introduce complex fuzzy soft matrices and define some new operations on these matrices. Moreover we develop an algorithm using complex fuzzy soft matrices and apply it to a decision making problem in signal processing.
\end{abstract}

Mathematics Subject Classification (2010). 03E72, 15B15

Keywords. complex fuzzy sets, complex fuzzy matrices

\section{Introduction}

Fuzzy set has been introduced by Zadeh in 1965 [14], which is nowadays used in almost all the branches of science. Basically, it is a suitable tool for modeling as the crisp models lack clarity while handling problems in different fields of science like artificial intelligence, computer science, control engineering, decision theory, expert system, logic management science, operations research, robotics, and many others. In other words this concept of fuzzy set is used to address those uncertain problems which arise in models representing real life phenomenon. This concept is not only limited to the above mentioned problems, rather it is also been used in business, medical, and related health sciences successfully. Maiers and Sherif [5] in their index of fuzzy set applications identified more then twelve subject areas including decision making, economics, engineering, and operations research on the basis of literature available on the subject.

In 2002, Ramot et al. [13] introduced a new concept of a complex fuzzy set which is the generalization of a fuzzy set whose range is not restricted to a closed interval $[0,1]$ but expanded to a unit circle in complex plane. It is defined as:

$$
\mu_{S}(x)=\gamma_{S}(x) e^{\iota \omega_{S}(x)}
$$

where $\gamma_{S}(x)$ is called amplitude of grade of membership belongs to $[0,1]$ and $\omega_{S}(x)$ is a real valued function. Thus $\mu_{S}(x)$ is a complex valued function that maps each input value to a value in unit circle in complex plane. The authors in this paper added one more dimension to the degree of membership. Thus the complex fuzzy set has a phase term in addition to its amplitude term. The phase term represents wave type characteristic of this

\footnotetext{
*Corresponding Author.

Email addresses: madadmath@yahoo.com (M. Khan), saimaanispk@gmail.com (S. Anis), szsong@jejunu.ac.kr (S.Z. Song), skywine@gmail.com (Y.B. Jun)

Received: 15.01.2018; Accepted: 14.02.2019
} 
set which makes it different from the basic concept of the fuzzy set. The idea of complex fuzzy set is the extended form of the fuzzy set.

In [13], Ramot et al., defined union, intersection, and complement on this set and explored several interesting properties and they gave real life application of this concept.

Complex fuzzy sets solved the issues raised in the problems that are very difficult or impossible to be represented by the traditional concept of the fuzzy sets.

N. Çağman and S. Enginoğlu introduced soft matrices and explore several properties of these matrices. They developed a soft max-min decision making algorithm which can be used as a powerful tool in the problems involving uncertainties [3]. Moreover they introduced fuzzy soft matrices along with a fs-max-min decision making method which can be used successfully in decision making problems that contain ambiguities [4]. In fact they gave a new direction for fundamental work as well as applications in the theory of soft sets and fuzzy soft sets. For more details about soft sets, complex fuzzy sets and fuzzy soft matrices see [1], [6-12], and [15].

In this paper we introduce complex fuzzy soft matrices with some new operations. We develop an algorithm using these matrices and apply it to a problem in signals and systems.

\section{Preliminaries}

Suppose that $U$ is an initial universe set and $E$ is a set of parameters, let $P(U)$ denote the power set of $U$. A pair $(F, E)$ is called a soft set over $U$ where $F$ is a mapping given by $F: E \rightarrow P(U)$. Clearly a soft set is a mapping from parameters to $P(U)$ and it is not a set, but a parameterized family of subsets of the universe.

Let $I=[0,1]$ and let us denote all the subintervals of $I$ by $[I]$

A function $A: X \rightarrow[I]$ is called an interval-valued fuzzy set (briefly, an IVF set) in a non-empty set $X$.

Definition 2.1. A complex fuzzy set is defined as [13]:

$$
\mu_{S}(x)=\gamma_{S}(x) e^{\imath \omega_{S}(x)},
$$

where $\gamma_{S}(x)$ is called amplitude of grade of membership belongs to $[0,1]$ and $\omega_{S}(x)$ is a real valued function.

Definition 2.2. Let $U$ be an initial universe set, $E$ be the set of parameters and $A \subset E$. Then a pair $\left(\Phi_{\mu}, A\right)$ is called fuzzy soft set over $U$, where $\Phi_{\mu}$ is a mapping given by $\Phi_{\mu}: A \rightarrow P\left(\mu_{U}\right)$, where $P\left(\mu_{U}\right)$ denote the set of all complex fuzzy subsets of $U$.

\section{Complex fuzzy soft matrix theory}

In this section we introduce a new concept of a complex fuzzy soft matrix.

Complex Fuzzy Soft Matrix: Let $U=\left\{c_{1}, c_{2}, c_{3}, \ldots, c_{n}\right\}$ be the universal set and $E$ be the set of parameters given by $E=\left\{e_{1}, e_{2}, e_{3}, \ldots, e_{m}\right\}$. If $A \subset E$, then $\left(\Phi_{\mu}, A\right)$ is a fuzzy soft matrix over $U$, where $\Phi_{\mu}$ is a mapping given by $\Phi_{\mu}: A \rightarrow P\left(\mu_{U}\right)$ and $\eta_{\Xi}: A \rightarrow C^{U}$ where $C^{U}$ denotes the set of all fuzzy subsets and $P\left(C^{U}\right)$ denotes the power set of $C^{U}$. Then complex fuzzy soft set $\left(\Phi_{\mu}, A\right)$ can be expressed in matrix form as $\left[\widetilde{A}_{m \times n}\right]=\left[\left|a_{i j}\right|\right]_{m \times n}$, for $i=1,2, \ldots, m$ and $j=1,2,3, \ldots, n$, where

$$
\left|a_{i j}\right|= \begin{cases}\left|\eta\left(e_{i}\right)\right|_{j} & \text { if } e_{i} \in A \\ 0 & \text { otherwise }\end{cases}
$$

$\left|\eta\left(e_{i}\right)\right|_{j} \quad\left(\left\{\eta\left(e_{i}\right)\right\}_{j}\right.$ is a complex fuzzy set $)$ represents the element of $\widetilde{A}$ corresponds to element $c_{j}$ of $U$, for $j=1,2,3, \ldots, n$, where $\left|\eta\left(e_{i}\right)\right|_{j}=\alpha_{R C}$ such that $\alpha \in[0,1]$ and $R=1,2,3, \ldots, m$ and $C=1,2,3, \ldots, n$.

Following is an example to illustrate the newly defined complex fuzzy soft matrix. 
Example 3.1. Suppose that there are three houses under consideration, namely the universes $U=\left\{h_{1}, h_{2}, h_{3}\right\}$, and the parameter set $E=\left\{e_{1}, e_{2}, e_{3}\right\}$, where $e_{i}$ stands for "near to downtown", "green", and "cheap " respectively. Consider the mapping $\Phi_{\mu}$ from parameter set $A=\left\{e_{1}, e_{2}\right\} \subset E$ to the set of all fuzzy subsets of power set $U$. Consider a fuzzy soft set $\left(\Phi_{\mu}, A\right)$ which describes the "attractiveness of houses" that is considering for purchase. Then fuzzy soft set $\left(\Phi_{\mu}, A\right)$ is given as.

$$
\begin{aligned}
{\left[\Phi_{\mu}, A\right] } & =\left[\left|\eta\left(e_{i}\right)\right|_{j}\right]_{m \times n}, \text { where } \\
\Phi_{\mu}\left(e_{1}\right) & =\left\{\left(h_{1},\left|0.2 e^{i \frac{\pi}{2}}\right|\right),\left(h_{2},\left|0.3 e^{i 2 \pi}\right|,\left(h_{3},\left|0.4 e^{i \frac{\pi}{4}}\right|\right)\right.\right. \\
\Phi_{\mu}\left(e_{2}\right) & =\left\{\left(h_{1},\left|0.6 e^{i \frac{\pi}{6}}\right|\right),\left(h_{2},\left|0.9 e^{i(0.5)}\right|\right),\left(h_{3},\left|0.1 e^{i(0)}\right|\right),\right.
\end{aligned}
$$

where

$$
\begin{aligned}
0.2 e^{i \frac{\pi}{2}}= & 0.2\left(\cos \frac{\pi}{2}+i \sin \frac{\pi}{2}\right)=0.2(0+i)=0.2 i \\
|0.2 i|= & \sqrt{0.04}=0.2 \\
0.3 e^{i 2 \pi}= & 0.3(\cos 2 \pi+i \sin 2 \pi)=0.3(1+0)=0.3 \\
|0.3|= & \sqrt{0.09}=0.3 . \\
0.4 e^{i \frac{\pi}{4}}= & 0.4\left(\cos \frac{\pi}{4}+i \sin \frac{\pi}{4}\right)=0.4\left(\frac{1}{\sqrt{2}}+i \frac{1}{\sqrt{2}}\right) \\
= & 0.4(0.707+i 0.707)=0.28+i 0.28 \\
|0.28+i 0.28| & =\sqrt{0.078+0.078}=0.16 \\
0.6 e^{i \frac{\pi}{6}} & =0.6\left(\cos \frac{\pi}{6}+i \sin \frac{\pi}{6}\right)=0.6(0.87+0.5 i) \\
& =0.52+0.3 i \\
|0.52+0.3 i| & =\sqrt{0.27+0.09}=0.6 \\
0.9 e^{i(0.5)}= & 0.9(\cos 0.5+i \sin 0.5)=0.9(0.99+0.009 i) \\
= & 0.891+0.081 i \\
|0.891+0.081 i| & \sqrt{0.793+0.006}=0.89 \\
0.1 e^{i(0)} & =0.1(\cos 0+i \sin 0)=0.1 \\
\mid 0.1 e^{i(0)} & =0.1
\end{aligned}
$$

We would represent this complex fuzzy soft set in matrix form as:

$$
\left(\begin{array}{ccc}
0.2 & 0.6 & 0 \\
0.3 & 0.89 & 0 \\
0.16 & 0.1 & 0
\end{array}\right)
$$

Definition 3.2. Let $\left[a_{i j}\right]$ be a complex fuzzy soft matrix. Then $\left[a_{i j}^{\tilde{A}}\right]$ is called complex zero soft matrix if $\left(a_{i j}, r_{i j}\right)=(0,0)$ for all $i$ and $j$ and denoted by $\left[a_{i j}^{\tilde{A}}\right]=[0]$.

$\widetilde{A}_{m \times n}=\left[\left|a_{i j}\right|\right]_{m \times n}$, for $i=1,2, \ldots, m$ and $j=1,2,3, \ldots, n$, where

$$
\left|a_{i j}\right|= \begin{cases}\left|\eta\left(e_{i}\right)\right|_{j} & \text { if } e_{i} \in A \\ 0 & \text { otherwise }\end{cases}
$$

$\left|\eta\left(e_{i}\right)\right|_{j}$ represents the element of $\widetilde{A}$ corresponds to the element $c_{j}$ of $U$, for $j=1,2,3, \ldots, n$, where $\left|\eta\left(e_{i}\right)\right|_{j}=\alpha_{R C}$ such that $\alpha \in[0,1]$ and $R=1,2,3, \ldots, m$ and $C=1,2,3, \ldots, n$. 
Definition 3.3. Let $\left[\widetilde{A}_{m \times n}\right]$ and $\left[\widetilde{B}_{m \times n}\right]$ be complex fuzzy soft matrices. Then

(i) $\left[\widetilde{A}_{m \times n}\right]$ is a complex fuzzy soft submatrices of $\left[\widetilde{B}_{m \times n}\right]$, denoted by $\left[\widetilde{A}_{m \times n}\right] \sqsubseteq\left[\widetilde{B}_{m \times n}\right]$, if $\left(\left|a_{i j}\right| \leq\left|b_{i j}\right|\right.$ for all $\left|a_{i j}\right| \in\left[\widetilde{A}_{m \times n}\right]$ and $\left|b_{i j}\right| \in\left[\widetilde{B}_{m \times n}\right]$.

(ii) $\left[\widetilde{A}_{m \times n}\right]$ is a proper complex fuzzy soft submatrices of $\left[\widetilde{B}_{m \times n}\right]$, denoted by $\left[\widetilde{A}_{m \times n}\right] \subset$ $\left[\widetilde{B}_{m \times n}\right]$, if $\left(\left|a_{i j}\right| \leq\left|b_{i j}\right|\right.$ for all $\left|a_{i j}\right| \in\left[\widetilde{A}_{m \times n}\right]$ and $\left|b_{i j}\right| \in\left[\widetilde{B}_{m \times n}\right], n$, and for at least one term $\left|a_{i j}\right|<\left|b_{i j}\right|$.

(iii) $\left[\widetilde{A}_{m \times n}\right]$ is an equal complex fuzzy soft matrix of $\left[\widetilde{B}_{m \times n}\right]$, denoted by $\left[\widetilde{A}_{m \times n}\right]=$ $\left[\widetilde{B}_{m \times n}\right]$, if $\left(\left|a_{i j}\right|=\left|b_{i j}\right|\right.$ for all $\left|a_{i j}\right| \in\left[\widetilde{A}_{m \times n}\right]$ and $\left|b_{i j}\right| \in\left[\widetilde{B}_{m \times n}\right]$.

Definition 3.4. Let $\left[\widetilde{A}_{m \times n}\right]$ and $\left[\widetilde{B}_{m \times n}\right]$ be complex fuzzy soft matrices. Then the complex fuzzy soft matrices $\left[\widetilde{C}_{m \times n}\right]$ are called

(i) union of $\left[\widetilde{A}_{m \times n}\right]$ and $\left[\widetilde{B}_{m \times n}\right]$, denoted $\left[\widetilde{A}_{m \times n}\right] \cup\left[\widetilde{B}_{m \times n}\right]$ if $\left[\widetilde{C}_{m \times n}\right]=\max \left\{\left|a_{i j}\right|,\left|b_{i j}\right|\right\}$ for all $\left|a_{i j}\right| \in\left[\widetilde{A}_{m \times n}\right]$ and $\left|b_{i j}\right| \in\left[\widetilde{B}_{m \times n}\right]$.

(ii) intersection of $\left[\widetilde{A}_{m \times n}\right]$ and $\left[\widetilde{B}_{m \times n}\right]$, denoted $\left[\widetilde{A}_{m \times n}\right] \cap\left[\widetilde{B}_{m \times n}\right]$ if $\left[\widetilde{C}_{m \times n}\right]=\min \left\{\left|a_{i j}\right|,\left|b_{i j}\right|\right\}$ for all $\left|a_{i j}\right| \in\left[\widetilde{A}_{m \times n}\right]$ and $\left|b_{i j}\right| \in\left[\widetilde{B}_{m \times n}\right]$.

(iii) complement of $\left[\widetilde{A}_{m \times n}\right]$ denoted by $\left[\widetilde{A}_{m \times n}\right]^{\circ}$, if $\widetilde{C}_{m \times n}=\tilde{1}-\widetilde{A}_{m \times n}$ for all $m$ and $n$.

Example 3.5. Assume that

$$
\left[\widetilde{A}_{m \times n}\right]=\left[\begin{array}{cccc}
0.2 & 0.6 & 0 & 0.3 \\
0.3 & 0.9 & 0 & 0.2 \\
0.9 & 0.1 & 0 & 0.4 \\
0.16 & 0.4 & 0 & 0.8
\end{array}\right] \text { and }\left[\widetilde{B}_{m \times n}\right]=\left[\begin{array}{cccc}
0.1 & 0.5 & 0 & 0.3 \\
0.3 & 0.8 & 0 & 0.2 \\
0.9 & 0.7 & 0 & 0.5 \\
0.4 & 0.4 & 0 & 0.6
\end{array}\right]
$$

Then

$$
\begin{aligned}
& {\left[\widetilde{A}_{m \times n}\right] \cup\left[\widetilde{B}_{m \times n}\right]=\left[\begin{array}{cccc}
0.2 & 0.6 & 0 & 0.3 \\
0.3 & 0.9 & 0 & 0.2 \\
0.9 & 0.7 & 0 & 0.5 \\
0.16 & 0.4 & 0 & 0.8
\end{array}\right],\left[\widetilde{A}_{m \times n}\right] \cap\left[\widetilde{B}_{m \times n}\right]=\left[\begin{array}{cccc}
0.1 & 0.5 & 0 & 0.3 \\
0.3 & 0.8 & 0 & 0.2 \\
0.9 & 0.1 & 0 & 0.4 \\
0.4 & 0.4 & 0 & 0.6
\end{array}\right] \text { and }} \\
& {\left[\widetilde{A}_{m \times n}\right]^{\circ}=\left[\begin{array}{cccc}
0.8 & 0.4 & 1 & 0.7 \\
0.7 & 0.1 & 1 & 0.8 \\
0.1 & 0.9 & 1 & 0.6 \\
0.84 & 0.6 & 1 & 0.2
\end{array}\right]}
\end{aligned}
$$

Definition 3.6. Let $\left[\widetilde{A}_{m \times n}\right]$ and $\left[\widetilde{B}_{m \times n}\right]$ be complex fuzzy soft matrices. Then $\left[\widetilde{A}_{m \times n}\right]$ and $\left[\widetilde{B}_{m \times n}\right]$ are disjoint if $\left[\widetilde{A}_{m \times n}\right] \cap\left[\widetilde{B}_{m \times n}\right]=[0]$ for all $m$ and $n$.

Proposition 3.7. Let $\left[\widetilde{A}_{m \times n}\right]$ be a complex fuzzy soft matrix. Then

(i) $\left(\left[\widetilde{A}_{m \times n}\right]^{\circ}\right)^{\circ}=\left[\widetilde{A}_{m \times n}\right]$,

(ii) $[0]^{\circ}=\tilde{1}$.

Proposition 3.8. If $\left[\widetilde{A}_{m \times n}\right]$, $\left[\widetilde{B}_{m \times n}\right]$ and $\left[\widetilde{C}_{m \times n}\right]$ are complex fuzzy soft matrices then

(i) $\left[\widetilde{A}_{m \times n}\right]=\left[\widetilde{B}_{m \times n}\right]$ and $\left[\widetilde{B}_{m \times n}\right]=\left[\widetilde{C}_{m \times n}\right] \Leftrightarrow\left[\widetilde{A}_{m \times n}\right]=\left[\widetilde{C}_{m \times n}\right]$.

(ii) $\left[\widetilde{A}_{m \times n}\right] \sqsubseteq\left[\widetilde{B}_{m \times n}\right]$ and $\left[\widetilde{B}_{m \times n}\right] \sqsubseteq\left[\widetilde{A}_{m \times n}\right] \Leftrightarrow\left[\widetilde{A}_{m \times n}\right]=\left[\widetilde{B}_{m \times n}\right]$.

Proposition 3.9. If $\left[\widetilde{A}_{m \times n}\right]$ and $\left[\widetilde{B}_{m \times n}\right]$ are complex fuzzy soft matrices then

(i) $\left[\widetilde{A}_{m \times n}\right] \sqsubseteq\left[\widetilde{B}_{m \times n}\right]$ and $\left[\widetilde{B}_{m \times n}\right] \sqsubseteq\left[\widetilde{C}_{m \times n}\right] \Rightarrow\left[\widetilde{A}_{m \times n}\right] \sqsubseteq\left[\widetilde{C}_{m \times n}\right]$.

(ii) $\left[\widetilde{A}_{m \times n}\right] \sqsubseteq\left[\widetilde{B}_{m \times n}\right] \Leftrightarrow\left[\widetilde{A}_{m \times n}\right] \cap\left[\widetilde{B}_{m \times n}\right]=\left[\widetilde{A}_{m \times n}\right] \Leftrightarrow\left[\widetilde{A}_{m \times n}\right] \cup\left[\widetilde{B}_{m \times n}\right]=\left[\widetilde{B}_{m \times n}\right]$. 
Proposition 3.10. If $\left[\widetilde{A}_{m \times n}\right]$ and $\left[\widetilde{B}_{m \times n}\right]$ are complex fuzzy soft matrices then

(i) $\left[\widetilde{A}_{m \times n}\right] \cup\left[\widetilde{B}_{m \times n}\right]=\left[\widetilde{B}_{m \times n}\right] \cup\left[\widetilde{A}_{m \times n}\right]$.

(ii) $\left[\widetilde{A}_{m \times n}\right] \cap\left[\widetilde{B}_{m \times n}\right]=\left[\widetilde{B}_{m \times n}\right] \cap\left[\widetilde{A}_{m \times n}\right]$.

(iii) $\left(\left[\widetilde{A}_{m \times n}\right] \cup\left[\widetilde{B}_{m \times n}\right]\right) \cup\left[\widetilde{C}_{m \times n}\right]=\left[\widetilde{A}_{m \times n}\right] \cup\left(\left[\widetilde{B}_{m \times n}\right] \cup\left[\widetilde{C}_{m \times n}\right]\right)$.

(iv) $\left(\left[\widetilde{A}_{m \times n}\right] \cap\left[\widetilde{B}_{m \times n}\right]\right) \cap\left[\widetilde{C}_{m \times n}\right]=\left[\widetilde{A}_{m \times n}\right] \cap\left(\left[\widetilde{B}_{m \times n}\right] \cap\left[\widetilde{C}_{m \times n}\right]\right)$.

(v) $\left[\widetilde{A}_{m \times n}\right] \cup\left(\left[\widetilde{B}_{m \times n}\right] \cap\left[\widetilde{C}_{m \times n}\right]\right)=\left(\left[\widetilde{A}_{m \times n}\right] \cup\left[\widetilde{B}_{m \times n}\right]\right) \cap\left(\left[\widetilde{A}_{m \times n}\right] \cup\left[\widetilde{C}_{m \times n}\right]\right)$.

(vi) $\left[\widetilde{A}_{m \times n}\right] \cap\left(\left[\widetilde{B}_{m \times n}\right] \cup\left[\widetilde{C}_{m \times n}\right]\right)=\left(\left[\widetilde{A}_{m \times n}\right] \cap\left[\widetilde{B}_{m \times n}\right]\right) \cup\left(\left[\widetilde{A}_{m \times n}\right] \cap\left[\widetilde{C}_{m \times n}\right]\right)$.

Proposition 3.11. Let $\left[\widetilde{A}_{m \times n}\right]$ and $\left[\widetilde{B}_{m \times n}\right]$ be complex fuzzy soft matrices. Then De Morgan law are valid

$$
\begin{aligned}
& \text { (i) }\left(\left[\widetilde{A}_{m \times n}\right] \cup\left[\widetilde{B}_{m \times n}\right]\right)^{\circ}=\left(\left[\widetilde{A}_{m \times n}\right]\right)^{\circ} \cap\left(\left[\widetilde{B}_{m \times n}\right]\right)^{\circ} . \\
& \text { (ii) }\left(\left[\widetilde{A}_{m \times n}\right] \cap\left[\widetilde{B}_{m \times n}\right]\right)^{\circ}=\left(\left[\widetilde{A}_{m \times n}\right]\right)^{\circ} \cup\left(\left[\widetilde{B}_{m \times n}\right]\right)^{\circ} .
\end{aligned}
$$

Proof. For all $m$ and $n$

(i) $\left(\left[\widetilde{A}_{m \times n}\right] \cup\left[\widetilde{B}_{m \times n}\right]\right)^{\circ}$

$$
\begin{aligned}
& =\left[\max \left(\left[\widetilde{A}_{m \times n}\right],\left[\widetilde{B}_{m \times n}\right]\right)\right]^{\circ} \\
& =\left[1-\max \left(\widetilde{A}_{m \times n}, \widetilde{B}_{m \times n}\right)\right] \\
& =\left[\min \left(1-\widetilde{A}_{m \times n}, 1-\widetilde{A}_{m \times n}\right)\right] \\
& =\left[\widetilde{A}_{m \times n}\right]^{\circ} \cap\left[\widetilde{B}_{m \times n}\right]^{\circ} .
\end{aligned}
$$

(ii) It can be proved similarly.

Note. It is worth mentioning to add here that a complex fuzzy soft matrix is more general than a fuzzy soft matrix since that both the degree of membership function and phase terms are added in each entry of the matrix yielding better choice in decision making problems.

\section{Decision making algorithm}

We are going to discuss a real life application of newly defined complex fuzzy soft matrix. In fact we will show that how our theoretical results have real life applications. Specifically the complex fuzzy soft matrix explains how to get better and clear signal for identification with given reference signal.

Definition 4.1. [2] Let $U=\left\{u_{1}, u_{2}, u_{3}, \ldots u_{n}\right\}$ be initial universal set and $M m\left(C_{k i}\right)=$ $\left(D_{i 1}\right)$. Then subset of $U$ can be obtained by using $\left[D_{i 1}\right]=\widetilde{o p t}\left[\left(\widetilde{d}_{i 1}, d_{i 1}\right)\right]$, where

$$
\widetilde{o p t}\left(\widetilde{d}_{i 1}\right)(U)=\left\{\widetilde{d}_{i 1} / u_{i}: u_{i} \in U\right\} \text { and } \operatorname{opt}\left(d_{i 1}\right)(U)=\left\{d_{i 1} / u_{i}: u_{i} \in U\right\},
$$

$\left\{\widetilde{o p t}\left(\widetilde{d}_{i 1}\right)\right.$, opt $\left.\left(d_{i 1}\right)(U)\right\}$ is called an optimum fuzzy set on $U$.

Step 1. If a receiver gets various signals $S_{1}(g), S_{2}(g), S_{3}(g), \ldots, S_{m}(g)$ from any source. Each signal is sampled $\mathrm{N}$ times by the receiver. Then $S_{i}(g)$ ( $i$ varies from 1 to $m$ ) signals can be recognized with respect to $R$, where $R$ is given known signal. Assume that both $S_{i}(g)$ and $R$ are considered as $n$ times. 
Assume that $S_{j}(t)$ is $i$-th signal, where $1 \leq t \leq m$. Then the absolute value of each $S_{j}(t)$ in terms of discrete complex fuzzy transform given as follows:

where $g_{j, n}$ is the complex Fourier coefficients of signals $S_{j}$, and $S_{j}(t)$ varies from 1 to $m$. The above expression with alternate complex Fourier coefficients can be written as:

where $g_{j, s}=u_{j, s} \cdot e^{i \beta_{j, s}}$ such that $u_{j, s}$ and $\beta_{j, s}$ are real valued and $u_{j, s} \geq 1$ for all $s$ $(1 \leq s \leq m)$.

Step 2. Expressed in matrix form as $\widetilde{A}_{m \times n}=\left[\left|S_{j}(t)\right|\right]_{N \times m}$, that is, in the matrix take all the signals in columns and each column contains $N$ samples of every signal, so we get

$$
A=\left[\begin{array}{cccc}
\left|S_{1}(1)\right| & \left|S_{2}(1)\right| & \ldots & \left|S_{m}(1)\right| \\
\left|S_{1}(2)\right| & \left|S_{2}(2)\right| & \ldots & \left|S_{m}(2)\right| \\
. & . & . & \dot{.} \\
\left|S_{1}(N)\right| & \left|S_{2}(N)\right| & \ldots & \left|S_{m}(N)\right|
\end{array}\right]
$$

Step 3. In same way make another matrix by the signals $S_{j}^{\prime}(t)(1 \leq t \leq m$ and $1 \leq j \leq N)$.

$$
B=\left[\begin{array}{cccc}
\left|S_{1}^{\prime}(1)\right| & \left|S_{2}^{\prime}(1)\right| & \ldots & \left|S_{m}^{\prime}(1)\right| \\
\left|S_{1}^{\prime}(2)\right| & \left|S_{2}^{\prime}(2)\right| & \ldots & \left|S_{m}^{\prime}(2)\right| \\
\cdot & \cdot & \cdot & \cdot \\
\left|S_{1}^{\prime}(N)\right| & \left|S_{2}^{\prime}(N)\right| & \ldots & \left|S_{m}^{\prime}(N)\right|
\end{array}\right]
$$

Step 4. Find usual product of the matrices.

Step 5. Find complex fuzzy soft max-min decision making matrix $(C S M m D M)$.

Step 6. Find optimum fuzzy set on $U$.

\subsection{Application}

Let us assume that the set of four signals $U=\left\{\eta_{1}, \eta_{2}, \eta_{3}, \eta_{4}\right\}$. Now each of these signals is sampled four times. Let $R$ be the given known reference signal. Each signal is compared with the reference signal in order to get the high degree of resemblance with the reference signal $R$. First use the (1), then we obatin the matrix $A$ by setting the signals along column and their four times sampling along row. Similarly we will obtain the matrix $B$.

Now on the basis of steps algorithm $(1-3)$ defined above we discuss an example.

$$
\begin{aligned}
\widetilde{A} & =\left(\begin{array}{llll}
0 & 0.1 & 0.2 & 0.2 \\
0 & 0.3 & 0.3 & 0.3 \\
0 & 0.2 & 0.2 & 0.4 \\
0 & 0.3 & 0.2 & 0.1
\end{array}\right) \\
\widetilde{B} & =\left(\begin{array}{llll}
0.1 & 0 & 0.1 & 0.2 \\
0.2 & 0 & 0.3 & 0.1 \\
0.3 & 0 & 0.2 & 0.4 \\
0.4 & 0 & 0.1 & 0.3
\end{array}\right) .
\end{aligned}
$$

Step 4. Now the product of fuzzy soft matrices $\widetilde{A}$ and $\widetilde{B}$ is given as:

$$
\widetilde{A} * \widetilde{B}=\left(\begin{array}{cccc}
0.16 & 0.0 & 0.09 & 0.15 \\
0.27 & 0.0 & 0.18 & 0.24 \\
0.18 & 0.0 & 0.14 & 0.22 \\
0.16 & 0.0 & 0.14 & 0.14
\end{array}\right)
$$

It is simple usual matrix multiplication. 
Step 5. To calculate $\left.M m[\widetilde{A} * \widetilde{B}]=\left[D_{i 1}\right]=\left[d_{i 1}\right)\right]$, we have to find $\left[D_{i 1}\right]$ for all $i \in$ $\{1,2,3,4\}$, such that

$$
d_{i 1}=\min \left\{t_{k 1}\right\}=\min \left\{t_{11}, t_{21}, t_{31}, t_{41}\right\}, \text { for } k \in\{1,2,3,4\} .
$$

Now let us calculate $\left[D_{11}\right]=\left(d_{11}\right)$ for fixed $j=1$.

$$
d_{11}=\min \left\{t_{k 1}\right\}=\min \left\{t_{11}, t_{21}, t_{31}, t_{41}\right\} .
$$

Here we have to find $\widetilde{t}_{k 1}$ for all $k \in\{1,2,3,4\}$ and $t_{k 1}$ for all $k \in\{1,2,3,4\}$. Let us find $\widetilde{t}_{k 1}$ for $k \in\{1,2,3,4\}$ as:

$$
t_{11}=0.16, t_{21}=0.27, t_{31}=0.18, t_{41}=0.16 .
$$

Now

$$
d_{11}=\min \{0.16,0.27,0.18,0.16\}=0.16
$$

So $\left(D_{11}\right)=\left(d_{11}\right)=(0.16)$.

Similarly we can find $\left(D_{21}\right)=\left(d_{21}\right),\left(D_{31}\right)=\left(d_{31}\right)$ and $\left(D_{41}\right)=\left(d_{41}\right)$, where

$$
d_{21}=\min \left\{t_{12}, t_{22}, t_{32}, t_{42}\right\}=\min \{0,0,0,0\}=0 .
$$

So $\left(D_{21}\right)=\left(d_{21}\right)=0$.

$$
d_{31}=\min \{0.09,0.18,0.14,0.14\}=0.09 .
$$

Therefore $D_{31}=d_{31}=0.09$.

Also

$$
d_{41}=\min \{0.15,0.24,0.22,0.14\}=0.14
$$

So $\left(D_{41}\right)=d_{41}=0.14$.

Finally we can obtain fuzzy soft max-min decision fuzzy soft matrix as:

$$
\begin{aligned}
m M(\widetilde{A} * \widetilde{B})= & \left(D_{i 1}\right)=\left(d_{i 1}\right) \\
= & \left(\begin{array}{l}
\left(D_{11}\right)=\left(d_{11}\right) \\
\left(D_{21}\right)=\left(d_{21}\right) \\
\left(D_{31}\right)=\left(d_{31}\right) \\
\left(D_{41}\right)=\left(d_{41}\right)
\end{array}\right)=\left(\begin{array}{l}
0.16 \\
0.00 \\
0.09 \\
0.14
\end{array}\right) .
\end{aligned}
$$

Step 6. Finally we can find an optimum fuzzy set on $[U]=(u)$.

$$
\widetilde{\text { opt Mm }}(A * B)(\eta)=\left\{0.16 / \eta_{1}, 0.09 / \eta_{3}, 0.14 / \eta_{4}\right\} .
$$

Hence identify signal $\eta_{1}$ as $R$.

\section{Conclusion}

In this paper, we introduced the concept of a complex fuzzy soft matrix and defined different types of matrices in fuzzy soft set theory along with examples. Then we introduced some new operations on these matrices and explored related properties. Further we constructed a complex fuzzy soft decision making model, designed an algorithm with the help of these matrices, and used it in decision making problems. We hope that our finding will help enhancing the study on fuzzy soft set theory and will open a new direction for applications especially in decision analysis.

Acknowledgment. The first two authors are grateful to Higher Education Commission of Pakistan for financial support. The third author (S.Z. Song) was supported by Basic Science Research Program through the National Research Foundation of Korea(NRF) funded by the Ministry of Education (No. 2016R1D1A1B02006812). 


\section{References}

[1] B. Chetia and P.K. Das, Some results of intuitionistic fuzzy soft matrix theory, Adv. Appl. Sci. Res. 3 (1), 412-423, 2012.

[2] N. Çağman and S. Enginoğlu, Soft set theory and uni-int decision making, Eur. J. Oper. Res. 207 (2), 848-855, 2010.

[3] N. Çağman and S. Enginoğlu, Soft matrix theory and its decision making, Comput. Math. Appl. 59, 3308-3314, 2010.

[4] N. Çağman and S. Enginoğlu, Fuzzy soft matrix theory and its applications in decision making, Iran J. Fuzzy Syst. 9 (1), 109-119, 2012.

[5] J. Maiers and Y.S. Sherif, Applications of fuzzy set theory, IEEE Trans. Syst. Man Cybern. 15 (1), 175-189, 1985.

[6] D. Molodstov, Soft set theory first-results, Comput. Math. Appl. 37 (4-5), 190-31, 1999.

[7] S. Mondal and M. Pal, Soft matrices, Afr. J. Math. Comput. Sci. Res. 4 (13), 379-388, 2011.

[8] P.K. Maji, R. Biswas, and A.R. Roy, Fuzzy soft sets, J. Fuzzy Math. 9 (3), 589-502, 2001.

[9] S. Miodrag and L.D. Petković, Complex interval arithmetic and its applications, John Wiley \& Sons, 1998.

[10] P. Rajarajeswari and P. Dhanalakshmi, An application of interval valued intuitionistic fuzzy soft matrix theory in medical diagnosis, to appear in Ann. Fuzzy Math. Inform.

[11] A.K. Shyamal and M. Pal, Interval-valued fuzzy matrices, J. Fuzzy Math. 14 (3), 583-604, 2006.

[12] Y. Yang and J. Chenli, Fuzzy soft matrices and their applications, Lect. Notes Comput. Sc. (Part I) 7002, 618-627, 2011.

[13] D. Ramot, R. Milo, M. Friedman, and A. Kandel, Complex fuzzy sets, IEEE Trans. Fuzzy Syst. 10 (2), 171-186, 2012.

[14] L.A. Zadeh, Fuzzy sets, Inform. Control 8, 338-353, 1965.

[15] G. Zhang, T.S. Dillon, K.-Y. Cai, J. Ma, and J. Lu, Operation properties and $\delta$ equalities of complex fuzzy sets. Int. J. Approx. Reason. 50, 1227-1249, 2009. 